\title{
A singular perturbation problem with a turning point
}

\author{
A.M. Watts \\ We consider the equation

$$
\varepsilon y^{\prime \prime}+p(x) y^{\prime}+q(x) y=0,
$$ \\ where $\varepsilon$ is a small positive parameter and $p$ vanishes in the \\ interval. Two asymptotic forms of solution are obtained and a \\ rigorous estimate is made of the difference between the exact \\ solutions and the asymptotic forms.
}

\section{Introduction}

The equation

$$
\varepsilon y^{\prime \prime}+p(x) y^{\prime}+q(x) y=0,
$$

where $\varepsilon$ is a small positive parameter, has been studied extensively and the asymptotic properties of the solutions are well understood when $p$ does not change sign. Cases when $p$ has a simple zero have been considered by Ackerberg and O'Malley [1], but they have made the assumption that $p$ and $q$ are analytic functions. Here we show that quite general results can be obtained for the solution in the interval $0 \leq x \leq 1$ under the much weaker conditions that $q$ be continuously differentiable and $p$ twice continuously differentiable. We assume $p(0)=0, p^{\prime}(0)=-1$ and $p$ is strictly negative for $0<x \leq I$. We use the notation $q(0)=b$. The continuation of the solutions through the origin is also considered.

Interest in this equation developed from the related problem of the solution of the von Kármán similarity equations for a rotating fluid. The

Received 7 January 1971. 
angular velocity satisfies an equation of the same form as (1.1) with the parameter $\varepsilon$ corresponding to the inverse of the Reynold's number. The change in sign of the coefficient $p$ corresponds to a reversal of the axial velocity in the fluid, which is usually associated with a change in sign of the angular velocity. The understanding of the flow between counter-rotating discs is the eventual aim of this work, but it has not yet been achieved.

Some preliminary forms of approximate solutions are obtained in order to explain the choice of variables used below. Near the origin the approximate equation is

$$
\varepsilon y^{\prime \prime}-x y^{\prime}+b y=0
$$

With $\eta=x \varepsilon^{-\frac{1}{2}}$, we obtain

$$
\frac{d^{2} y}{d n^{2}}-n \frac{d y}{d n}+b y=0 .
$$

The solutions of this equation are the parabolic cylinder functions, which also play an important role in the solution of the approximate equation for larger values of the independent variable.

Away from the origin, the outer or slowly varying approximate solution is given by

$$
p(x) y^{\prime}+q(x) y=0 .
$$

The solution of this equation is

$$
Y_{0}(x)=x^{b} \exp \left\{-\int_{0}^{x}\left(\frac{q(\tau)}{p(\tau)}+\frac{b}{\tau}\right) d \tau\right\} .
$$

To obtain the rapidly varying boundary layer solution, we use a change of variable to

$$
t=-\frac{1}{\varepsilon} \int_{0}^{x} p(\tau) d \tau
$$

Equation (1.1) becomes

$$
\frac{d^{2} y}{d t^{2}}-\frac{d y}{d t}+\varepsilon\left(q-p^{\prime}\right) y=0
$$

and the approximate solution is 


$$
y=A e^{t}
$$

It must be remembered that since $t$ is a stretched variable this solution is valid only over a small interval, that is, over an interval in which $x$ changes by a small quantity. The important consequence of this solution for our purposes is that the variable $t$ is an appropriate one for the rapidly varying solution, while $x$ is appropriate for the slowly varying solution. We note also that for $x \rightarrow 0,2 t \sim \frac{x^{2}}{\varepsilon}=\eta^{2}$, which suggests that $t^{\frac{1}{2}}$ might be a more suitable variable to use.

More useful forms of approximate solutions valid over the whole interval $0 \leq x \leq 1$ are derived in the next section.

\section{Construction of approximate solutions}

The independent variable $\xi(x)$ which is suggested by the preliminary forms of the approximate solutions is given by

$$
\varepsilon \xi^{2} \equiv X^{2}=-2 \int_{0}^{x} p(\tau) d \tau
$$

Let

$$
\xi_{1}=\xi(1) .
$$

Equation (1.1), in terms of $\xi$, becomes

$$
\text { (2.3) } \begin{aligned}
L(y) \equiv \frac{d^{2} y}{d \xi^{2}}+P(\xi ; \varepsilon) \frac{d y}{d \xi}+Q(\xi ; \varepsilon) y & \equiv \frac{d^{2} y}{d \xi^{2}}-\xi \frac{d y}{d \xi}+b y \\
& -\varepsilon^{\frac{1}{2}}\left(\frac{1}{X}+\frac{p^{\prime} X}{p^{2}}\right) \frac{d y}{d \xi}+\left(b-\frac{X^{2} q}{p^{2}}\right) y=0 .
\end{aligned}
$$

As before $b=q(0), p^{\prime}(0)=-1$ and from (2.1) we have

$$
X \sim x \text { for } x \rightarrow 0 \text {, }
$$

so that

$$
b-\frac{X^{2} q}{p^{2}}+0 \text { as } x \rightarrow 0,
$$

that is 


$$
b-\frac{X^{2} q}{p^{2}} \sim C x \text { for } x \rightarrow 0
$$

for some constant $C$, since $q, p, X$ are continuously differentiable functions of $x$.

$$
\text { Similarly, }\left(\frac{x}{X}+\frac{p^{\prime} X x}{p^{2}}\right) \text { vanishes at } x=0 \text { and, since } p^{\prime}, p \text { and } X
$$

are continuously differentiable functions of $x$, we have that $\left(\frac{1}{X}+\frac{p^{\prime} X}{p^{2}}\right)$ is bounded.

A "two-variable" method is used to determine the approximate solutions. These are of the form

$$
w(\xi ; \varepsilon)=\alpha(x) u(\xi),
$$

where

$$
u^{\prime \prime}-\xi u^{\prime}+b u=0,
$$

and the derivatives of $w$ are given by formulae such as

$$
w^{\prime}=\alpha u^{\prime}+\frac{d x}{d \xi} \alpha^{\prime} u \text {. }
$$

For the sake of abbreviation we write

$$
w(\xi) \equiv w(\xi ; \varepsilon) \text {. }
$$

Substitution into $(2.3)$ gives

$$
\text { (2.6) } \begin{aligned}
L(\alpha u)=\varepsilon^{\frac{1}{2}}\left[\frac{2 X \alpha^{\prime}}{p}+\left(\frac{1}{X}+\frac{p^{\prime} X}{p^{2}}\right) \alpha\right] u^{\prime}- & {\left[\frac{X^{2} \alpha^{\prime}}{p}-\left(b-\frac{X^{2} q}{p^{2}}\right) \alpha\right] u } \\
& -\varepsilon\left[\frac{X^{2} \alpha^{\prime \prime}}{p^{2}}-\frac{X^{2} p^{\prime} \alpha^{\prime}}{p^{3}}-\frac{\alpha^{\prime}}{p}+\frac{X \alpha^{\prime}}{p}\right] u .
\end{aligned}
$$

There is no $\alpha$ which would make this expression vanish, but we may choose $\alpha$ so that the expression obtained when $u$ and $u^{\prime}$ are replaced by their asymptotic forms vanishes. If we were to use an iterative method to find more accurate approximate solutions, this choice of $\alpha$ would prevent the development of "resonance" in the next approximation.

We derive two approximate solutions $w_{1}, w_{2}$ where

$$
w_{1}(\xi)=\alpha_{1}(x) u_{1}(\xi), \quad w_{2}(\xi)=\alpha_{2}(x) u_{2}(\xi) .
$$


$u_{1}$ and $u_{2}$ are solutions of $(2.4)$, chosen so that

$$
\begin{aligned}
& u_{1}=c_{1} \xi^{b}\left(1+o\left(\xi^{-2}\right)\right), \\
& u_{2}=c_{2} \xi^{-b-1} e^{\frac{1}{2} \xi^{2}}\left(1+o\left(\xi^{-2}\right)\right), \text { for } \xi \rightarrow \infty .
\end{aligned}
$$

The asymptotic forms of the derivatives of $u_{1}$ and $u_{2}$ may be shown to be

$$
\begin{aligned}
& u_{1}^{\prime}=b c_{1} \xi^{b-1}\left(1+o\left(\xi^{-2}\right)\right), \\
& u_{2}^{\prime}=c_{2} \xi^{-b} e^{\frac{1}{2} \xi^{2}}\left(1+o\left(\xi^{-2}\right)\right) .
\end{aligned}
$$

$u_{1}$ and $u_{2}$ are scaled so that their Wronskian is $e^{\frac{1}{2} \xi^{2}}$.

With $u$ replaced by $u_{1}$, and $\alpha$ by $\alpha_{1}$, the term in equation (2.6) which dominates is

$$
\left[\frac{X^{2} \alpha_{1}^{\prime}}{p}-\left(b-\frac{X^{2} q}{p^{2}}\right) \alpha_{1}\right] u_{1} .
$$

To make this vanish, $\alpha_{1}$ is chosen to be

$$
\alpha_{1}(x)=X^{-b} y_{0}(x) \text {, }
$$

where $Y_{0}$ is the outer solution given by equation (1.4). Note that $Y_{0}$ has the factor $x^{b}$ so that $\alpha_{1}$ is bounded and non-zero at the origin as well as over the remainder of the interval. Also, $X$ and $Y_{0}$ are twice continuously differentiable and so is $\alpha_{1}$, provided we make the further restrictions that $p$ is three times continuously differentiable and $q$ twice, in the neighbourhood of $x=0$.

With $u_{2}$ and $\alpha_{2}$, the dominant terms in (2.6) are

$$
\text { (2.11) } \begin{aligned}
\varepsilon^{\frac{1}{2}}\left[\frac{2 X \alpha_{2}^{\prime}}{p}+\left(\frac{1}{X}+\frac{p^{\prime} X}{p^{2}}\right) \alpha_{2}\right] u_{2}^{\prime}-\left[\frac{X^{2} \alpha_{2}^{\prime}}{p}-\left(b-\frac{X^{2} q}{p^{2}}\right) \alpha_{2}\right] u_{2} \\
=\left[\frac{X^{2}}{p} \alpha_{2}^{\prime}+\left(b+1+\frac{p^{\prime} X^{2}}{p^{2}}-\frac{X^{2} q}{p^{2}}\right) \alpha_{2}\right] u_{2}+o\left(\xi^{-2}\right) u_{2} .
\end{aligned}
$$

$\alpha_{2}$ is then given by

$$
\alpha_{2}^{\prime}+\left(\frac{(b+1) p}{x^{2}}+\frac{p^{\prime}}{p}-\frac{q}{p}\right) \alpha_{2}=0,
$$


from which we obtain

$$
\alpha_{2}=\frac{x^{b+1}}{p Y_{0}}
$$

and again we note that $\alpha_{2}$ has two continuous derivatives for $0 \leq x \leq 1$, and does not vanish in the interval, under the same conditions as $\alpha_{1}$.

We now have two approximate solutions $\omega_{1}$ and $w_{2}$ with

(2.12) $L\left(w_{1}\right)=\varepsilon^{\frac{1}{2}} g_{1}(x) u_{1}^{\prime}(\xi)+\varepsilon f_{1}(x) u_{1}=F_{1}(\xi)$,

(2.13) $L\left(w_{2}\right)=\varepsilon^{\frac{1}{2}} g_{2}(x) u_{2}^{\prime}(\xi)+h_{2}(x) u_{2}(\xi)+\varepsilon f_{2}(x) u_{2}(\xi)=E_{2}(\xi)$,

where

$$
\begin{aligned}
& g_{1}(x)=\frac{2 X \alpha_{1}^{\prime}}{p}+\left(\frac{1}{X}+\frac{p^{\prime} X}{p^{2}}\right) \alpha_{1}, \\
& f_{1}(x)=-\left[\frac{X^{2} \alpha_{1}^{\prime \prime}}{p^{2}}-\frac{X^{2} p^{\prime} \alpha_{1}^{\prime}}{p^{3}}-\frac{\alpha_{1}^{\prime}}{p}+\frac{X \alpha_{1}^{\prime}}{p}\right], \\
& h_{2}(x)=-\left[\frac{X^{2} \alpha_{2}^{\prime}}{p}-\left(b-\frac{X^{2} q}{p^{2}}\right) \alpha_{2}\right],
\end{aligned}
$$

and $g_{2}(x), f_{2}(x)$ are defined in a similar way to $g_{1}, f_{1}$ but with $\alpha_{1}$ replaced by $\alpha_{2}$.

It is clear that the functions $g_{1}, g_{2}, f_{1}, f_{2}$ and $h_{2}$ are all bounded and $O(1)$ for $\varepsilon \rightarrow 0$. We also have, since $h_{2}$ is continuously differentiable and vanishes at the origin,

$$
\left|h_{2}(x)\right|<K x
$$

for some constant $K$.

Further, because of our choice of $\alpha_{2}$, we have

$$
\left|\varepsilon^{\frac{1}{2}} g_{2} u_{2}+h_{2} u_{2}\right|<K \xi^{-2} u_{2}(\xi)
$$

for $\xi>\xi_{0}$, where $\xi_{0}$ is some number independent of $\varepsilon$ and greater than the largest zero of $u_{2}$.

Note that the quantities on the left-hand sides of (2.17), (2.18) may be simultaneously small since $x$ may be small while $\xi$ is large. 


\section{Existence of solutions}

Our task now is to show that, in the interval $0 \leq \xi \leq \xi_{1}$, there exist exact solutions of (2.3) to which $w_{1}$ and $w_{2}$ are approximations. This is done by the construction of a suitable integral equation.

Since $w_{1}$ and $w_{2}$ are twice differentiable functions of $\xi$, there is a second order linear operator of the form

$$
M(w)=w^{\prime \prime}+P_{1}(\xi, \varepsilon) w^{\prime}+Q_{1}(\xi, \varepsilon) w,
$$

such that

$$
M\left(w_{1}\right)=M\left(w_{2}\right)=0 .
$$

Equations (2.12), (2.13) may be written in the form

$$
\begin{aligned}
& w_{1}^{\prime \prime}+P w_{1}^{\prime}+Q w_{1}=F_{1}, \\
& w_{2}^{\prime \prime}+P w_{2}^{\prime}+Q w_{2}=F_{2},
\end{aligned}
$$

and we have, by our definition of the operator $M$,

$$
\begin{aligned}
& w_{1}^{\prime \prime}+P_{1} \omega_{1}^{\prime}+Q_{1} w_{1}=0, \\
& w_{2}^{\prime \prime}+P_{1} \omega_{2}^{\prime}+Q_{1} \omega_{1}=0 .
\end{aligned}
$$

These two pairs of equations give

$$
\begin{aligned}
& \left(P-P_{1}\right) w_{1}^{\prime}+\left(Q-Q_{1}\right) w_{1}=F_{1}, \\
& \left(P-P_{1}\right) w_{2}^{\prime}+\left(Q-Q_{1}\right) w_{2}=F_{2},
\end{aligned}
$$

so that

$$
\begin{aligned}
& P_{2} \equiv P-P_{1}=-\frac{w_{2} F_{1}+w_{1} F_{2}}{W\left(w_{1}, w_{2}\right)}, \\
& Q_{2} \equiv Q-Q_{1}=\frac{w_{2}^{\prime} F_{1}-w_{1} F_{2}}{W\left(w_{1}, w_{2}\right)},
\end{aligned}
$$

where $W\left(w_{1}, w_{2}\right)$ is the Wronskian of $w_{1}, w_{2}$.

Equation (2.3) may be expressed in the form

$$
M(y(\xi ; \varepsilon))=-P_{2} y^{\prime}-Q_{2} y .
$$

In view of (3.2) we can use $w_{1}$ and $w_{2}$ to construct a suitable Green's function for the operator $M$ and obtain an integral equation equivalent to (3.5) The appropriate integral equation is 


$$
y(\xi)=A w_{1}(\xi)+B w_{2}(\xi)+T y(\xi),
$$

where $T$ is the integral operator given by

$$
\text { (3.7) } \begin{aligned}
T y(\xi)=-w_{1}(\xi) \int_{0}^{\xi}[W(n)]^{-2} w_{2}(n)\{ & {\left[w_{1}(n) F_{2}(n)-w_{2}(n) F_{1}(n)\right] y^{\prime}(n) } \\
& \left.+\left[w_{2}^{\prime}(n) F_{1}(n)-w_{1}^{\prime}(n) F_{2}(n)\right] y(n)\right\} d n \\
- & w_{2}(\xi) \int_{\xi}^{\xi_{1}}[W(n)]^{-2} w_{1}(n)\left\{\left[w_{1}(n) F_{2}(n)-w_{2}(n) F_{1}(n)\right] y^{\prime}(n)\right. \\
+ & {\left.\left[w_{2}^{\prime}(n) F_{1}(n)-w_{1}^{\prime}(n) F_{2}(n)\right] y(n)\right\} d n, }
\end{aligned}
$$

where

$$
W(n)=W\left(w_{1}(n), w_{2}(n)\right),
$$

and $\xi_{1}$ is defined by (2.2).

Let $v_{1}(\xi), v_{2}(\xi)$ be two continuous functions defined by

$$
\begin{aligned}
& v_{1}(0)=v_{2}(0)=1, \\
& v_{1}^{\prime}(\xi)=v_{2}^{\prime}(\xi)=1 \text { for } 0 \leq \xi \leq \xi_{0}, \\
& v_{1}^{\prime}(\xi)=\xi^{b-1}, \\
& v_{2}^{\prime}(\xi)=\xi^{-b} e^{\frac{1}{2} \xi^{2}} \text { for } \xi_{0} \leq \xi \leq \xi_{1} .
\end{aligned}
$$

We consider the function spaces $S_{1}$ and $S_{2}$, where $S_{1}$ consists of functions satisfying, for some constant $K$ which depends on the function,

$$
|f(\xi)|<K v_{1}(\xi),\left|f^{\prime}(\xi)\right|<K v_{1}^{\prime}(\xi),
$$

and similarly, $S_{2}$ consists of the differentiable functions satisfying

$$
|f(\xi)|<K v_{2}(\xi),\left|f^{\prime}(\xi)\right|<K v_{2}^{\prime}(\xi) .
$$

After some lengthy calculations using (2.17), (2.18), we can show that $T$ is a contractive mapping in both $S_{1}$ and $S_{2}$ with contraction factors that are $O\left(\varepsilon^{\frac{1}{3}}\right)$, and hence that there exist exact solutions $y_{1}, y_{2}$ of equation (2.3) with 


$$
\begin{aligned}
& y_{1}(\xi ; \varepsilon)=w_{1}(\xi)+o\left(\varepsilon^{\frac{1}{3}}\right) v_{1}(\xi), \\
& y_{1}^{\prime}(\xi ; \varepsilon)=w_{1}^{\prime}(\xi)+o\left(\varepsilon^{\frac{1}{3}}\right) v_{1}^{\prime}(\xi), \\
& y_{2}(\xi ; \varepsilon)=w_{2}(\xi)+o\left(\varepsilon^{\frac{1}{3}}\right) v_{2}(\xi), \\
& y_{2}^{\prime}(\xi ; \varepsilon)=w_{2}^{\prime}(\xi)+o\left(\varepsilon^{\frac{1}{3}}\right) v_{2}^{\prime}(\xi) .
\end{aligned}
$$

\section{Continuation of solutions through the origin}

One of the most important extensions of the problem originally posed is the continuation of the solutions through the zero of the coefficient $p$. Clearly, with similar conditions on the coefficients, similar results can be obtained for negative $x$ as for positive $x$. We need to relate the asymptotic forms of solution for negative $x$ to those for positive $x$.

Suppose firstly that $b$ is neither zero nor a positive integer. Then a reference to Miller [3, pp. 687, 689], for example, tells us that the solution $u_{1}(\xi)$ of (2.4) satisfying the first of (2.8) is exponentially large for large negative $\xi$, that is

$$
u_{1}=C_{3}(-\xi)^{-b-1} e^{\frac{1}{2} \xi^{2}}\left\{1+o\left(\xi^{-2}\right)\right\} \text { for } \xi \rightarrow-\infty \text {. }
$$

Similarly $u_{2}(\xi)$ may be chosen to satisfy (2.4), the second of (2.8) and

$$
u_{2}=c_{4}(-\xi)^{b}\left(1+o\left(\xi^{-2}\right)\right) \text { for } \xi \rightarrow-\infty,
$$

so that the roles of $u_{1}$ and $u_{2}$ are reversed on passing through the origin. Let two approximate solutions for $x<0$ be $\beta_{1}(x) u_{1}(\xi)$, $\beta_{2}(x) u_{2}(\xi)$, where $\beta_{1}$ and $\beta_{2}$ are chosen in the same manner as $\alpha_{2}$ and $\alpha_{1}$, respectively. Suppose also that $\beta_{1}(0)=\alpha_{1}(0), \beta_{2}(0)=\alpha_{2}(0)$.

$$
\text { Let } z_{1}(\xi ; \varepsilon), z_{2}(\xi ; \varepsilon) \text { be exact solutions of }(2.3) \text { to which } \beta_{1} u_{1}
$$
and $B_{2} u_{2}$ approximate. By (3.9) and similar equations for $z_{1}, z_{2}$ we have 


$$
\begin{aligned}
& y_{1}(0 ; \varepsilon)=\alpha_{1}(0) u_{1}(0)+O\left(\varepsilon^{\frac{1}{3}}\right)=z_{1}(0 ; \varepsilon)+O\left(\varepsilon^{\frac{1}{3}}\right), \\
& y_{1}^{\prime}(0 ; \varepsilon)=\alpha_{1}(0) u_{1}^{\prime}(0)+O\left(\varepsilon^{\frac{1}{3}}\right)=z_{1}^{\prime}(0 ; \varepsilon)+O\left(\varepsilon^{\frac{1}{3}}\right), \\
& y_{2}(0 ; \varepsilon)=z_{2}(0 ; \varepsilon)+O\left(\varepsilon^{\frac{1}{3}}\right), \\
& y_{2}^{\prime}(0 ; \varepsilon)=z_{2}^{\prime}(0 ; \varepsilon)+O\left(\varepsilon^{\frac{1}{3}}\right) .
\end{aligned}
$$

Clearly, $y_{1}$ and $y_{2}$ may be continued into negative values of $\xi$, and $z_{1}$ and $z_{2}$ may each be expressed as a linear combination of $y_{1}$ and $y_{2}$. Using their relations at the origin we have

$$
\begin{aligned}
& \text { (4.1) } y_{1}(\xi ; \varepsilon)=z_{1}(\xi ; \varepsilon)+O\left(\varepsilon^{\frac{1}{3}}\right) z_{1}(\xi ; \varepsilon)+O\left(\varepsilon^{\frac{1}{3}}\right) z_{2}(\xi ; \varepsilon), \\
& \text { (4.2) } y_{2}(\xi ; \varepsilon)=z_{2}(\xi ; \varepsilon)+O\left(\varepsilon^{\frac{1}{3}}\right) z_{1}(\xi ; \varepsilon)+O\left(\varepsilon^{\frac{1}{3}}\right) z_{2}(\xi ; \varepsilon) .
\end{aligned}
$$

In terms of the prototype function $v_{2}$,

$$
y_{1}(\xi ; \varepsilon)=z_{1}(\xi ; \varepsilon)+o\left(\varepsilon^{\frac{1}{3}}\right) v_{2}(-\xi) \text {. }
$$

We cannot use $z_{2}$ or $B_{2} \mathfrak{U}_{2}$ as an approximation to $y_{2}$ for $\xi<0$, since the term $O\left(\varepsilon^{\frac{1}{3}}\right) z_{1}(\xi ; \varepsilon)$ on the right of (4.2) dominates for large negative $\xi$. However, in this case we can continue $z_{2}$ to positive values of $\xi$ and obtain, for $\xi>0$,

$$
z_{2}(\xi ; \varepsilon)=y_{2}(\xi ; \varepsilon)+O\left(\varepsilon^{\frac{1}{3}}\right) v_{2}(\xi) .
$$

Thus there are two solutions $y_{1}, z_{2}$ with $y_{1}$ exponentially large for negative $x$ and $z_{2}$ exponentially large for positive $x$.

These and their approximations allow us to obtain solutions to two-point boundary value problems where the conditions are applied on opposite sides of the origin. The solutions give a boundary layer at each boundary point with exponentially small values in the interior.

In the case when $b$ is a positive integer, $u_{1}(\xi)$ is a polynomial, while $u_{2}(\xi)$ is exponentially large for both large positive and large negative $\xi$. Equations (4.1) and (4.2) still hold but $y_{1}$ cannot be approximated by $z_{1}$ (or $B_{1} u_{1}$ ) because the term $O\left(\varepsilon^{\frac{1}{3}}\right) z_{2}(\xi ; \varepsilon$ ) on the right of (4.1) dominates for large negative $\xi$. Without the ability to extend the approximate form for $y_{1}$ through the origin we cannot find approximate solutions to two-point boundary value problems such as those 
discussed by Ackerberg and O'Malley [1] in Part 3 of their paper.

A useful example here is the equation

$$
\varepsilon y^{\prime \prime}-x y^{\prime}+(b+x) y=0 \text {. }
$$

The transformations

$$
\begin{gathered}
\xi=\frac{x}{\varepsilon^{\frac{1}{2}}}, \\
y=e^{\frac{\xi^{2}}{4}} z,
\end{gathered}
$$

yield

$$
\frac{d^{2} z}{d \xi^{2}}+\left[b+\varepsilon+\frac{1}{2}-\left(\frac{\xi-2 \varepsilon^{\frac{1}{2}}}{2}\right)^{2}\right] z=0,
$$

whose solutions are parabolic cylinder functions. If $b+\varepsilon$ is a positive integer, then there is a solution of (4.5) which is only algebraically large for both positive and negative $x$. If $b$ is a positive integer, any solution of (4.5) is exponentially large for positive $x$ or negative $x$ or both. This contradicts the results in Parts $3 \mathrm{a}, 3 \mathrm{~b}$ of the paper by Ackerberg and O'Malley.

\section{Remarks}

For an interval extending to $\infty$, the approximate solutions $w_{1}$ and $w_{2}$ are not valid, in that they do not approximate to the exact solutions $y_{1}$ and $y_{2}$. It is the terms containing $f_{1}(x)$ and $f_{2}(x)$ in (2.12), (2.13) which cause the trouble and it is these terms, as shown by (2.6), which contain $\alpha_{1}^{\prime \prime}$ and $\alpha_{2}^{\prime \prime}$. To obtain approximate solutions valid over larger intervals than $\left[0, \xi_{1}\right]$, it would be necessary to continue the two-variable process to higher order terms. The same type of behaviour is shown in problems of non-linear oscillations and in problems involving adiabatic invariants, where it is possible to trade off accuracy in the approximation for a larger interval of validity. That is, the approximate solutions become less accurate as the interval is extended until, after a sufficiently large time, the errors become comparable with the solution itself. There are, however, examples such as the asymptotic solutions of Bessel's equation where the first approximation to the solution is valid 
over an infinite interval.

The method used here of deriving approximate solutions by a two-variable procedure and then using them to obtain an integral equation is also applicable in problems which have been dealt with by other methods such as those described by Erdélyi [2] and Mitropol'skiY [4].

The restrictions on $p$ and $q$ that were introduced in Section 2 (after (2.10)) may be avoided by the use of a messy modification to the method. The third continuous derivative of $p$ and the second of $q$ are necessary only to ensure that $\alpha_{1}$ and $\alpha_{2}$ have bounded second derivatives in the neighbourhood of the origin, but the particular forms of $\alpha_{1}$ and $\alpha_{2}$ are chosen to satisfy asymptotic conditions for large $\xi$. Instead of adding these extra conditions on $p$ and $q$, we could use alternative approximate solutions given by

$$
\begin{aligned}
w_{1}(\xi) & =\alpha_{1}(x) u_{1}(\xi) & \xi_{0} & <\xi<\xi_{1}, \\
& =\beta_{1}(\xi ; \varepsilon) u_{1}(\xi) & 0 & <\xi<\xi_{0}, \\
w_{2}(\xi) & =\alpha_{2}(x) u_{2}(\xi) & \xi_{0} & <\xi<\xi_{1}, \\
& =\beta_{2}(\xi ; \varepsilon) u_{2}(\xi) & 0 & <\xi<\xi_{0},
\end{aligned}
$$

where $\beta_{1}$ and $\beta_{2}$ are chosen so that $\beta_{1}, \frac{d \beta_{1}}{d x}, \frac{d^{2} \beta_{1}}{d x^{2}}, \beta_{2}, \frac{d \beta_{2}}{d x}, \frac{d^{2} \beta_{2}}{d x^{2}}$ have the same values as $\alpha_{1}, \alpha_{1}^{\prime}, \alpha_{1}^{\prime \prime}, \alpha_{2}, \alpha_{2}^{\prime}, \alpha_{2}^{\prime \prime}$, respectively, at $\xi_{0}$. It is also specified that $\frac{d^{2} \beta_{1}}{d x^{2}}, \frac{d^{2} \beta_{2}}{d x^{2}}$ are $O\left(\varepsilon^{-\frac{1}{2}}\right)$, which is possible since $\alpha_{1}^{\prime \prime}$ and $\alpha_{2}^{\prime \prime}$ are of this order at $\xi=\xi_{0}$.

\section{References}

[1] R.C. Ackerberg and R.E. O'Malley, Jr, "Boundary layer problems exhibiting resonance", Studies in App 2. Math. 49 (1970), 277-295.

[2] A. Erdélyi, "On a nonlinear boundary value problem involving a small parameter", J. Austral. Math. Soc. 2 (1961/1962), 425-439. 
[3] J.C.P. Miller, "Parabolic cylinder functions", Handbook of mathematical functions with formulas, grophs and mathematical tables, 685-720 (edited by Milton Abramowitz; I rene A. Stegun, National Bureau of Standards Applied Mathematics Series 55; United States Department of Commerce, 1964; reprinted, Dover, New York, 1965).

[4] Yu. A. Mitropol'skii, Problems of the asymptotic theory of nonstationary vibrations (translated from the Russian by Ch. Gutfreund; Israel Program for Scientific Translations, Jerusalem, 1965).

Department of Mathematics, University of Queensland, St Lucia, Queensland. 